\title{
Structural and dynamical modeling of WINGS clusters
}

\section{The distribution of cluster galaxies of different morphological classes within regular and irregular clusters}

\author{
A. Cava ${ }^{1}$, A. Biviano ${ }^{2}$, G. A. Mamon ${ }^{3}$, J. Varela ${ }^{4}$, D. Bettoni ${ }^{5}$, M. D’Onofrio ${ }^{6}$, \\ G. Fasano ${ }^{5}$, J. Fritz ${ }^{7}$, M. Moles ${ }^{4}$, A. Moretti ${ }^{5}$, and B. Poggianti ${ }^{5}$ \\ ${ }^{1}$ Department of Astronomy, University of Geneva, 51 Ch. des Maillettes, 1290 Versoix, Switzerland \\ e-mail: antonio.cava@unige.ch \\ 2 INAF-Osservatorio Astronomico di Trieste, via Tiepolo 11, 34143 Trieste, Italy \\ 3 Institut d'Astrophysique de Paris, UMR 7095: CNRS \& UPMC Sorbonne-Universités, 98bis Bd Arago, 75014 Paris, France \\ ${ }^{4}$ Centro de Estudios de Física del Cosmos de Aragón, Plaza San Juan 1, 44001 Teruel, Spain \\ 5 INAF-Osservatorio Astronomico di Padova, Vicolo dell'Osservatorio 5, 35122 Padova, Italy \\ 6 Dipartimento di Fisica e Astronomia, Università degli Studi di Padova, Vicolo dell'Osservatorio 3, 35122 Padova, Italy \\ 7 Instituto de Radioastronomía y Astrofísica, UNAM, Campus Morelia, AP 3-72, CP 58089, Mexico
}

Received 14 March 2017 / Accepted 16 July 2017

\begin{abstract}
Context. We study the distribution of galaxies in nearby clusters to shed light on the evolutionary processes at work within clusters and prepare for a full dynamical analysis to be conducted in forthcoming papers of this series.

Aims. We use the Wide-field Nearby Galaxy-clusters Survey (WINGS) database complemented with literature data. We assign galaxy membership to individual clusters, then select a sample of 67 clusters with at least 30 spectroscopic members each. 53 of these clusters do not show evidence of substructures in phase-space, as measured by the Dressler-Shectman test, while 14 do. We estimate the virial radii and circular velocities of the 67 clusters by a variety of proxies (velocity dispersion, X-ray temperature, and richness) and use these estimates to build stack samples from these 53 and 14 clusters, that we call "Reg" and "Irr" stacks, respectively. We show that our results are robust with regard to the choice of the virial radii and circular velocities used to scale galaxy radii and velocities in the stacking procedure. We determine the number-density and velocity-dispersion profiles of Elliptical (E), S0, and Spiral+Irregular (S) galaxies in the Reg and Irr samples, separately, and fit models to these profiles.

Methods. The number density profiles of E, S0, and S galaxies are adequately described by either a Navarro, Frenk, \& White (NFW) or a cored King model, both for the Reg and Irr samples, with a slight preference for the NFW model. The spatial distribution concentration increases from the S to the S0 and to the E populations, both in the Reg and the Irr stacks, reflecting the well-known morphology-radius relation. Reg clusters have a more concentrated spatial distribution of E and S0 galaxies than Irr clusters, while the spatial distributions of S galaxies in Reg and Irr clusters have a similar concentration. We propose a new phenomenological model that provides acceptable fits to the velocity dispersion profile of all our galaxy samples. The velocity dispersion profiles become steeper and with a higher normalization from E to S0 to S galaxies. The S0 velocity dispersion profile is close to that of E galaxies in Reg clusters, and intermediate between those of $\mathrm{E}$ and $\mathrm{S}$ galaxies in Irr clusters.

Results. Our results suggest that $\mathrm{S}$ galaxies are a recently accreted cluster population, that take $\leq 3$ Gyr to evolve into S0 galaxies after accretion, and in doing so modify their phase-space distribution, approaching that of cluster ellipticals. While in Reg clusters this evolutionary process is mostly completed, it is still ongoing in Irr clusters.
\end{abstract}

Key words. galaxies: clusters: general - galaxies: kinematics and dynamics - galaxies: structure

\section{Introduction}

Clusters of galaxies have long been recognized as valuable tools for the study of cosmology and galaxy formation. They are the most massive virialized objects in the Universe and, as such, they sample the rarest density fluctuations in the Universe, which makes them excellent cosmological probes. They also provide excellent laboratories for the study of the influence of environment on galaxy evolution, on morphological evolution in particular. A useful tool in this respect is the analysis of the spatial and velocity distribution of cluster galaxies of different morphological types. In fact, a galaxy morphological type can be affected by physical processes whose efficiency depends both on the local density of galaxies or intra-cluster diffuse hot gas, and on the galaxy peculiar velocity within the cluster (see, e.g., Biviano 2011 , for a review of these processes).

In particular, the tidal damage (and stripping) consequent to galaxy-galaxy collisions is stronger for low-velocity encounters, eventually leading to a merger, and these encounters are more frequent in high galaxy-density environments (Spitzer \& Baade 1951). Tidal effects and mergers can transform the morphological type of a galaxy, most likely destroying or thickening disks and creating bulges (Barnes 1990). Repeated high-speed encounters can have similar effects on not too massive galaxies (Moore et al. 1996). The interaction of a galaxy with the cluster gravitational potential can lead to tidal truncation, depending on how close the galaxy ventures to the cluster center (Ghigna et al. 1998). Ram-pressure stripping can remove the gas content of a 
galaxy, and this process is more efficient in regions of high intracluster gas density, and for galaxies moving at high speed in the cluster (Gunn \& Gott 1972). Gas removal is believed to favor the transformation from $\mathrm{S}$ to $\mathrm{S} 0$.

There is strong observational evidence for morphological segregation of cluster galaxies, both with respect to local density and clustercentric distance, and with respect to velocity. In nearby clusters, Spiral and Irregular galaxies (S, hereafter) are known to live in regions of lower local density (Dressler 1980) and more distant from the cluster center (Sanromà \& Salvador-Solé 1990; Whitmore et al. 1993) than elliptical galaxies (E, hereafter), with $\mathrm{S} 0$ galaxies (S0, hereafter) displaying an intermediate spatial distribution between $\mathrm{S}$ and $\mathrm{E}$ galaxies. Early-type Spirals live in regions of intermediate local density between S0 and late-type Spirals (Thomas \& Katgert 2006a). Different cluster galaxy populations also display different distributions in velocity space, the velocity dispersion of E and S0 being smaller than that of S (Tammann 1972; Moss \& Dickens 1977; Sodré et al. 1989). Early-type Spirals have a velocity dispersion closer to S0 than to late-type Spirals (Adami et al. 1998). Combining the spatial and velocity distribution in the projected phase-space (PPS hereafter), Biviano et al. (2002) showed that it is possible to distinguish three main cluster galaxy populations, E+S0, early Spirals, and late Spirals.

At redshift $z \approx 0.5$, similar trends of morphology with density and clustercentric radius were found in clusters (Postman et al. 2005; Smith et al. 2005), but not in irregular ones (Dressler et al. 1997). The morphological content of clusters changes rapidly up to $z \approx 0.5$, with a decreasing fraction of S0 and an increasing fraction of S with $z$ (Dressler et al. 1997; Fasano et al. 2000; Postman et al. 2005; D'Onofrio et al. 2015), but then this evolution slows down at higher $z$ (Desai et al. 2007). This change is stronger in lower-mass clusters (Poggianti et al. 2009). The cluster velocity dispersion is mildly dependent on the morphological fraction (Desai et al. 2007), not only in low- $z$ but also in high- $z$ clusters, up to $z \approx 1$ at least.

Fasano et al. (2015) have recently revisited the morphologydensity paradigm, using the WIde-field Nearby Galaxy-cluster Survey (WINGS) database of galaxies in nearby clusters. They considered 5504 spectroscopically confirmed members of 76 nearby clusters, down to an absolute magnitude limit $M_{V}=$ -19.5. Fasano et al. (2015) found that the morphology-radius relation remains valid at all densities, while the morphologydensity relation is valid only in the inner cluster regions, and outside the inner regions only for very regular clusters without substructures. This result does not depend on the cluster mass, nor on the galaxy stellar mass. Galaxies of higher stellar mass were found to display a stronger dependence of their morphological type with density (but not with radius).

In this paper we extend the work of Fasano et al. (2015) by analyzing not only the spatial, but also the velocity distributions of WINGS cluster member galaxies of three morphological classes, E, S0, and S. Based on the results of Fasano et al. (2015), we use the clustercentric distance, rather than the local density, to parametrize the spatial distribution of different galaxy types. The morphological segregation in clustercentric distance is analyzed by comparison of the number density profiles (NDPs) of the three morphological classes. In line with previous studies (e.g., Adami et al. 1998; Desai et al. 2007), the morphological segregation in velocity is analyzed by comparison of the velocity dispersion profiles (VDPs) of the three morphological classes. To take full advantage of the large statistical sample of spectroscopically confirmed WINGS cluster members, we build two stacks of the best-sampled WINGS clusters, one for the regular (REG) and another for the irregular (IRR) clusters. The cluster regularity is based on the presence of substructures detected with the method of Dressler \& Shectman (1988, DS method hereafter). We will use the REG stack sample and some of the results obtained in this paper in future papers addressing the dynamics of the regular WINGS clusters (Mamon et al., in prep.; Biviano et al., in prep.).

The structure of this paper is the following. We present the data sample in Sect. 2. In Sect. 3 we describe our procedure to stack the data from different clusters. The PPS distributions (PPSD hereafter) are also presented separately for E, S0, and S in the REG and IRR stacks. In Sects. 4 and 5 we determine the NDPs and, respectively, the VDPs, separately for E, S0, and S, in the REG and IRR stacked clusters, to compare the relative spatial and velocity distributions of these different morphological galaxy classes in clusters of different dynamical status. Our results are discussed in Sect. 6 and we provide our summary and conclusions in Sect. 7. Throughout this paper we assume a $\Lambda$ CDM cosmological model with $\Omega_{\Lambda}=0.7, \Omega_{0}=0.3$, and $H_{0}=70 \mathrm{~km} \mathrm{~s}^{-1} \mathrm{Mpc}^{-1}$.

\section{The data}

\subsection{The WINGS database}

WINGS (Fasano et al. 2006) is an all-sky $(|b|>20)$ survey of a complete, X-ray selected sample of galaxy clusters in the redshift range $0.04-0.07$. The goal of the WINGS project is the systematic study of the local cosmic variance of the cluster population and of the properties of cluster galaxies as a function of cluster properties and local environment. The core of the WINGS project is the optical $(B, V)$ imaging survey (called WINGS-OPT; Varela et al. 2009). It provides photometric data for large samples of galaxies $(\sim 550000)$ and stars $(\sim 190000)$ in the inner field $\left(34^{\prime} \times 34^{\prime}\right)$ of 76 nearby galaxy clusters, as well as structural and morphological information for a subsample ( 40000$)$ of relatively bright galaxies (Pignatelli et al. 2006; Fasano et al. 2012; D’Onofrio et al. 2014). Additional photometric information comes from follow-up imaging surveys in the near-infrared (NIR) $J$ and $K$ bands (WINGS-NIR, 28 clusters; Valentinuzzi et al. 2009) and the $U$ Johnson band (17 clusters; Omizzolo et al. 2014). Currently, the photometric data set is being expanded by very wide field observations (four times the original WINGS area) taken with the VLT Survey Telescope (VST) OmegaCAM in the usual $B$ and $V$ Johnson bands (Gullieuszik et al. 2015) and in the Sloan $u$ band (D'Onofrio et al., in prep.).

The WINGS database is made available to the community through the Virtual Observatory (Moretti et al. 2014). WINGS is currently the only data set providing a homogeneous database of detailed photometric, morphological, and spectroscopic characteristics for several thousand galaxies in galaxy clusters.

\subsection{Spectroscopic data: the extended (WINGS+literature) sample}

Spectroscopic information for $\sim 6000$ galaxies in the field of 48 WINGS clusters has been provided by a follow-up multifibre, medium-resolution survey (WINGS-SPE; Cava et al. 2009). Detailed information about spectral features are available for a subset of these galaxies (Fritz et al. 2007, 2011, 2014). Follow-up spectroscopy of a subset of the WINGS clusters has been obtained using the Anglo Australian Telescope (AAT) AAOmega spectrograph (Moretti et al. 2017), to extend 
A. Cava et al.: Structural and dynamical modeling of WINGS clusters. I.

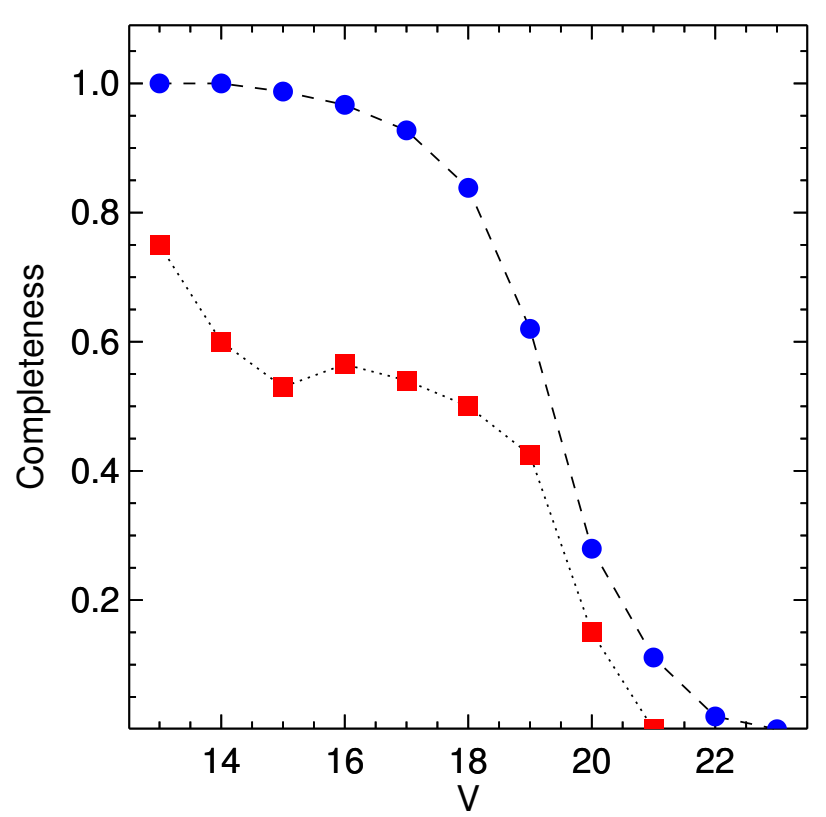

Fig. 1. Comparison of the $V$-band spectroscopic completeness for the original WINGS-SPE catalog presented in Cava et al. (2009; red squares and dotted line; including 48 clusters, 6137 galaxies) and for the current WINGS+literature (blue dots and dashed line; 73 clusters, 10277 galaxies) catalog.

the spectroscopic coverage of the WINGS clusters in their outer regions. Detailed morphological information is not yet available for the AAT/AAOmega additional sample, so this is not considered in the present study.

We refer to Cava et al. (2009, hereafter Paper I) for a detailed description of the WINGS-SPE spectroscopic survey, including a comparison with literature data. In this work we exploit the full dataset, WINGS and literature data, to perform a detailed dynamical analysis of the whole WINGS-OPT sample, including 76 galaxy clusters (cluster A3562 is excluded because $V$-band images were obtained under poor seeing conditions). In Paper I we have already presented a detailed data quality analysis and comparison with literature data, detailing the method for including literature data in the WINGS-SPE catalog (see also Moretti et al. 2014). After updating the catalog with the Sloan Digital Sky Survey (SDSS) DR7 and the latest NASA/IPAC Extragalactic Database (NED) data, our final catalog includes $\sim 10000$ galaxies with redshift determinations ( $\sim 60 \%$ from WINGS only), increasing the global spectroscopic completeness level (defined as the number counts of galaxies with redshifts to the number counts of galaxies in the photometric parent sample in magnitude bins, see Paper I) for our sample of galaxy clusters from $\sim 50 \%$ to $\sim 80 \%$ for galaxies below magnitude $\sim 18$, and reaching $50 \%$ completeness at a magnitude of 19.3. The completeness improvement is remarkable, as shown in Fig. 1. In Paper I, we compared multiple measurements of galaxy velocities to estimate their average external error. We find a rms $\sigma_{\Delta v} \sim 90 \mathrm{~km} \mathrm{~s}^{-1}$ in the worst case (see Fig. 4 and Table 5 of Paper I). This is larger than the average internal error estimate $\left(\delta v \sim 45-50 \mathrm{~km} \mathrm{~s}^{-1}\right.$ ), but still small enough not to affect the measurement of the internal velocity dispersion of galaxy clusters.

Our final spectroscopic sample contains 10288 galaxies in the field of 76 galaxy clusters from the WINGS-OPT sample. The average number of cluster members with redshifts is 93, rising to 105 for the clusters in the WINGS-SPE sample, and lowering to 70 for the clusters outside the WINGS-SPE sample.
This difference reflects the higher level of completeness attained by the WINGS multi-object spectroscopic observations. In the following we concentrate on the subsample of 73 clusters with $\geq 10$ galaxies with measured spectroscopic redshifts each, for a total of 10277 galaxies.

We complement our sample with the photometric and morphological information from the WINGS database (Moretti et al. 2014). Galaxy morphologies have been determined using the MORPHOT automatic tool of Fasano et al. (2012), which is optimized for the WINGS survey. It applies maximum likelihood and neural network methods to derive the best set of morphological parameters, and the equivalent morphological index, $T_{\mathrm{M}}$ (see Table 1 in Fasano et al. 2012, for a comparison between MORPHOT and visual classification indices). Adopting the MORPHOT classification, we define the three subsamples of ellipticals ("E"), with $T_{\mathrm{M}} \leq-4$, lenticulars ("S0"), with $-4<T_{\mathrm{M}} \leq 0$, and spirals + irregulars ("S"), with $T_{\mathrm{M}}>0$.

We finally assign a local completeness value to each galaxy in our sample. We estimate the completeness of the spectroscopic sample by comparing the number counts of galaxies with redshifts to the number counts of galaxies in the photometric sample in magnitude bins. These counts are estimated in radial bins around the cluster center, hence our completeness is a function of both the galaxy magnitude and its radial distance from the cluster center (see Moretti et al. 2014, Eq. (2)). In our completeness estimates we also take into account that there are regions where there was no observation at all due to geometrical constraints of the detectors, for example, gaps or asymmetric positioning of the CCDs, and of the brightest cluster galaxy (BCG) with respect to the field center. Completeness corrections are required to correctly estimate the intrinsic NDPs of the tracers of the gravitational potential from the observed spatial distribution of cluster members (see Sect. 4).

\section{The stacked samples of clusters}

\subsection{Definition of the stacks}

The first step in our analysis is the cluster membership determination. We adopt the "Clean" method of Mamon et al. (2013) to define cluster members for each cluster in the spectroscopic parent sample based on the projected phase-space $\left(R, v_{z}\right)$ distribution. We call $R$ (respectively $r$ ) the projected (respectively 3D) radial distance from the cluster center, that we identify with the position of the BCG.

The rest-frame line-of-sight (LOS) velocity is defined as $v_{z}=$ $c(z-\bar{z}) /(1+\bar{z})$, where $z$ is the galaxy redshift, $c$ is the speed of light, and $\bar{z}$ is the mean cluster redshift, estimated at each new iteration of the membership determination.

The Clean method works as follows:

1. look for gaps in the sorted velocity distribution using the gapper technique of Wainer \& Thissen (1976) with $C=4$ as proposed by Girardi et al. (1993);

2 . estimate the virial radius and velocity $\left(r_{\mathrm{v}}\right.$ and $v_{\mathrm{v}}$, respectively) from the aperture velocity dispersion $\left(\sigma_{\text {ap }}\right)$ computed with the robust median absolute deviation estimator, using a scaling of $\sigma_{\mathrm{ap}} / v_{\mathrm{v}}$ derived for Navarro, Frenk, \& White (1996, NFW hereafter) models with velocity anisotropy estimated in Mamon et al. (2010);

3 . filter the galaxies, keeping those within the estimated virial radius, $R<r_{\mathrm{v}}$, and within 2.7 predicted line-of-sight velocity dispersions for the NFW model with the Mamon et al. (2010) velocity anisotropy from the median velocity, $\left|v_{z}-\bar{v}_{z}\right|<$ $2.7 \sigma_{z}^{\mathrm{NFW}}(R)$; 
4. estimate the virial radius and velocity from the standard unbiased estimator of the aperture velocity dispersion within the previous estimated virial radius;

5. iterate on items 3-4 until convergence on membership.

For a subset (50 clusters) of the WINGS parent cluster sample, also X-ray temperatures, $T_{\mathrm{X}}$, are available (extracted from the $\mathrm{BAX}^{1}$ database). We provide alternative estimates of the virial radii using the mass-temperature scaling relation obtained from X-ray observations by Arnaud et al. (2005). We call $r_{200, X}$ these virial radius estimates.

Yet another possibility is to use a richness-based estimate of the virial radius (named "Num"; Mamon et al., in prep.; Old et al. 2014). This is obtained by selecting cluster members in a rectangular area of projected phase space within a projected radius of $1 \mathrm{Mpc}$ and an absolute line-of-sight velocity of $\pm 1333 \mathrm{~km} \mathrm{~s}^{-1}$ from the cluster center in projected space and velocity. The richness is defined as the total number of members within this rectangular section of PPS, corrected for radial incompleteness. We calibrate the richness-based virial radius estimates using a robustly determined relation between the logarithm of the richness and the $\sigma_{\mathrm{ap}}$-based $\log r_{200, \sigma}$, and we refer to this richness-based estimate of the virial radius as $r_{200, N}$.

In the end, we have three estimates of the virial radius:

1. $r_{200, \sigma}$ or "sigv" from the cluster aperture velocity dispersion;

2. $r_{200, \mathrm{X}}$ or "temp" from the cluster X-ray temperature;

3. $r_{200, N}$ or "Num" from the richness;

to which correspond three estimates of the circular velocity $v_{200, \sigma}, v_{200, \mathrm{X}}$, and $v_{200, N}$, respectively. For clusters without a X-ray temperature estimate, only the $r_{200, \sigma}$ and $r_{200, N}$ are available. In Table 1 we list the main properties of the clusters in our sample, including the available estimates of the virial radius.

We build three stack samples of clusters by rescaling the projected radial distances of cluster members by the cluster virial radii, $R_{n} \equiv R / r_{200}$, and the rest-frame velocities by the cluster virial velocities $v_{n} \equiv v_{z} / v_{200}$, one stack for each definition of $r_{200}, v_{200}$ (from sigv, Num, and temp).

On the stack sample we run again the Clean procedure for interloper removal. This is required because the Clean procedure can fail to reject some interlopers when the size of the individual cluster sample is not large enough. These remaining interlopers are, however, very few; less than $\sim 1 \%$ of the initially selected members are identified as interlopers by running the Clean procedure on the stack sample.

We do not consider all WINGS clusters in the stack, since some of them do not have a sufficient number of members $\left(N_{\mathrm{m}}\right.$ hereafter) for a reliable $r_{200}$ estimate. To choose the $N_{\mathrm{m}}$ limit for inclusion of a cluster in our sample, we have compared the values of $r_{200, \sigma}, r_{200, \mathrm{X}}$, and $r_{200, N}$. Decreasing $N_{\mathrm{m}}$ reduces the accuracy by which we can estimate $r_{200, \sigma}$ and $r_{200, N}$. In Fig. 2 we show the dispersion of the ratios $r_{200, \mathrm{X}} / r_{200, \sigma}$ (solid line), $r_{200, N} / r_{200, \sigma}$ (dashed line), and $r_{200, N} / r_{200, \mathrm{X}}$ (dash-dotted line) for samples of clusters with increasingly larger $N_{\mathrm{m}}$. Even if not strictly monotonic, there is a decreasing trend for the dispersion of $r_{200, \mathrm{X}} / r_{200, \sigma}$, that reaches a first (local) minimum at $N_{\mathrm{m}} \simeq 30$, and the absolute minimum at $N_{\mathrm{m}} \simeq 90$. The dispersion for the other $r_{200}$ ratios does not appear to depend on $N_{\mathrm{m}}$. We decide to consider the subsample of 68 clusters with $N_{\mathrm{m}} \geq 30$ and we check our results on a subsample of 38 clusters with $N_{\mathrm{m}} \geq 80$ (cutting at $N_{\mathrm{m}} \geq 90$ would leave only 28 clusters, which we

1 BAX is the X-ray galaxy clusters database: http://bax.ast. obs-mip.fr/

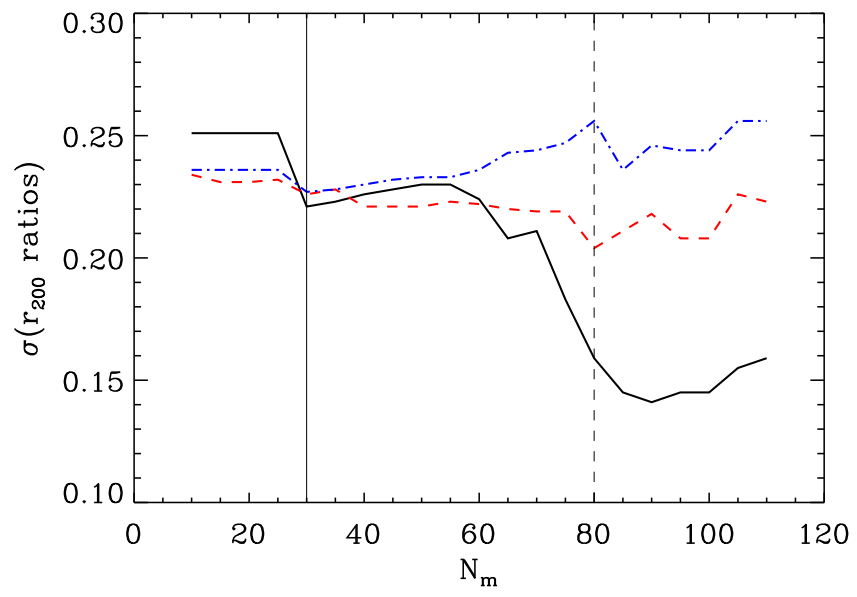

Fig. 2. Dispersion of the values of $r_{200, \mathrm{X}} / r_{200, \sigma}$ (black solid line), $r_{200, N} / r_{200, \sigma}$ (red dashed line) and $r_{200, N} / r_{200, \mathrm{X}}$ (blue dash-dotted line) for samples of clusters with increasingly larger number of members, $N_{\mathrm{m}}$.

consider too small a sample to be representative of the WINGS cluster population as a whole).

We run a 2D Kolmogorov-Smirnov (K-S) test (Fasano \& Franceschini 1987) to compare the PPSDs (the distributions in the $R_{n}, v_{n}$ space) of the $N_{\mathrm{m}} \geq 30$ and $N_{\mathrm{m}} \geq 80$ stack samples, and we find no statistically significant difference for any scaling (sigv, Num, or temp; $p \geq 0.1$ ). Hence in the following we only consider the $N_{\mathrm{m}} \geq 30$ stacks, as they contain a larger data set than the $N_{\mathrm{m}} \geq 80$ ones.

\subsection{Clusters with and without substructures}

We classify our sample into clusters with and without substructures, based on the statistical test introduced by Dressler \& Shectman (1988, DS hereafter). The DS test evaluates the mean velocity $\bar{v}_{\text {local }}$ and velocity dispersion $\sigma_{\mathrm{v}, \text { local }}$ of each group of $N_{\text {DS }}$ neighboring cluster members, and compares them with the corresponding quantities evaluated for the whole cluster. Rather than using the traditional DS method, we adopt here the modified version of the DS method suggested by Biviano et al. (2002; see also Cava 2008). In short, this modification consists in i) using $N_{\mathrm{DS}}=\sqrt{N_{\mathrm{m}}}$ rather than $N_{\mathrm{DS}}=11$, and ii) whenever a group has a velocity dispersion larger than the cluster global velocity dispersion, this group is not considered in the estimation of the global $\Delta$ parameter $^{2}$ (since the velocity dispersion is a proxy for the mass, this requirement means that we do not consider substructures that would be more massive than the cluster in which they are embedded).

In Table 1, we list the probability values of the DS statistic, $P_{\mathrm{DS}}$ (computed from 1000 resampling). We consider values $P_{\mathrm{DS}} \leq 0.05$ as significant evidence for substructure. Accordingly, we divide our sample of 68 clusters with $N_{\mathrm{m}} \geq 30$ into two subsamples, the regular - "Reg" - sample of 54 clusters with $P_{\mathrm{DS}}>0.05$, and the irregular - "Irr" - sample of 14 clusters with $P_{\mathrm{DS}} \leq 0.05$. From the two subsamples, we build six stacks, two for each of our three scalings (sigv, Num, and temp), on which we run the Clean algorithm to reject residual interlopers.

Using the 2D K-S test we compare the PPSDs (in normalized units, $R_{\mathrm{n}}, v_{\mathrm{n}}$ ) of the Reg and Irr stacks obtained using the three scalings and find no statistically different PPSDs (probability

2 The global $\Delta$ parameter is defined as the sum of the individual $\delta$ 's of all galaxies, where $\delta$ is defined in Eq. (1), Sect. 4.1 of Biviano et al. (2002). 
Table 1. WINGS cluster properties.

\begin{tabular}{|c|c|c|c|c|c|c|c|}
\hline Id & $\bar{z}$ & $N_{\mathrm{m}}$ & $f_{\mathrm{m}, \mathrm{vir}}$ & $r_{200, \sigma}$ & $r_{200, \mathrm{X}}$ & $r_{200, N}$ & $P_{\mathrm{DS}}$ \\
\hline A85 & 0.055 & 164 & 1.00 & 2.23 & 2.14 & 1.66 & 0.006 \\
\hline A119 & 0.044 & 175 & 1.00 & 1.87 & 2.01 & 1.78 & 0.030 \\
\hline A133 & 0.056 & 45 & 1.00 & 1.65 & 1.69 & 1.41 & 0.336 \\
\hline A147 & 0.044 & 21 & 1.00 & 1.42 & - & 1.31 & 0.913 \\
\hline A151 & 0.053 & 103 & 0.99 & 1.60 & - & 1.71 & 0.269 \\
\hline A160 & 0.044 & 81 & 1.00 & 1.52 & 0.99 & 1.60 & 0.324 \\
\hline A168 & 0.045 & 72 & 0.88 & 1.20 & 1.26 & 1.62 & 0.555 \\
\hline A193 & 0.048 & 73 & 1.00 & 1.60 & 1.24 & 1.59 & 0.840 \\
\hline A376 & 0.048 & 101 & 1.00 & 1.83 & 1.62 & 1.62 & 0.316 \\
\hline A500 & 0.068 & 92 & 0.99 & 1.57 & - & 1.70 & 0.358 \\
\hline A548b & 0.043 & 92 & 1.00 & 1.84 & - & 1.59 & 0.008 \\
\hline A602 & 0.060 & 51 & 1.00 & 1.49 & - & 1.40 & 0.345 \\
\hline A671 & 0.050 & 90 & 1.00 & 1.89 & 1.73 & 1.56 & 0.418 \\
\hline A754 & 0.055 & 230 & 1.00 & 2.23 & 2.48 & 1.84 & 0.138 \\
\hline A780 & 0.055 & 32 & 1.00 & 1.51 & 1.61 & 1.33 & 0.006 \\
\hline A957 & 0.045 & 88 & 1.00 & 1.52 & 1.31 & 1.52 & 0.096 \\
\hline A970 & 0.059 & 119 & 1.00 & 1.73 & 1.78 & 1.70 & 0.099 \\
\hline A1069 & 0.065 & 62 & 0.95 & 1.41 & - & 1.52 & 0.018 \\
\hline A1291 & 0.054 & 88 & 0.99 & 2.35 & 1.68 & 1.46 & 0.295 \\
\hline A1631a & 0.046 & 170 & 1.00 & 1.66 & 1.18 & 1.88 & 0.102 \\
\hline A1644 & 0.047 & 212 & 1.00 & 2.29 & 1.83 & 1.92 & 0.005 \\
\hline A1668 & 0.064 & 61 & 1.00 & 1.65 & - & 1.48 & 0.279 \\
\hline A1736 & 0.046 & 86 & 1.00 & 1.78 & 1.36 & 1.60 & 0.038 \\
\hline A1795 & 0.063 & 125 & 0.98 & 1.68 & 2.07 & 1.67 & 0.101 \\
\hline A1831 & 0.063 & 78 & 1.00 & 2.65 & - & 1.39 & 0.001 \\
\hline A1983 & 0.045 & 92 & 0.98 & 1.08 & 1.15 & 1.58 & 0.382 \\
\hline A1991 & 0.058 & 74 & 0.84 & 1.17 & 1.95 & 1.54 & 0.064 \\
\hline A2107 & 0.042 & 77 & 1.00 & 1.30 & 1.70 & 1.58 & 0.020 \\
\hline A 2124 & 0.066 & 83 & 0.96 & 1.67 & 1.76 & 1.50 & 0.703 \\
\hline A2149 & 0.065 & 35 & 0.43 & 0.83 & - & 1.26 & 0.959 \\
\hline A2169 & 0.058 & 55 & 0.69 & 1.09 & - & 1.43 & 0.020 \\
\hline A2256 & 0.058 & 197 & 0.74 & 2.88 & 2.22 & 1.41 & 0.000 \\
\hline A2271 & 0.057 & 10 & 1.00 & 0.98 & - & 1.45 & 0.044 \\
\hline A2382 & 0.064 & 169 & 1.00 & 1.86 & - & 1.77 & 0.098 \\
\hline A2399 & 0.058 & 146 & 0.98 & 1.52 & 1.22 & 1.76 & 0.039 \\
\hline A2415 & 0.058 & 100 & 0.99 & 1.52 & 1.37 & 1.74 & 0.688 \\
\hline A 2457 & 0.059 & 79 & 0.97 & 1.38 & - & 1.69 & 0.188 \\
\hline A $2572 a$ & 0.039 & 39 & 1.00 & 1.28 & 1.35 & 1.41 & 0.061 \\
\hline A2589 & 0.041 & 60 & 1.00 & 1.82 & 1.48 & 1.52 & 0.527 \\
\hline A 2593 & 0.041 & 106 & 1.00 & 1.43 & 1.41 & 1.75 & 0.180 \\
\hline A2622 & 0.061 & 46 & 1.00 & 1.74 & - & 1.57 & 0.807 \\
\hline A2626 & 0.057 & 76 & 1.00 & 2.64 & 1.34 & 1.54 & 0.900 \\
\hline A2657 & 0.040 & 29 & 0.93 & 0.88 & 1.61 & 1.37 & 0.842 \\
\hline A 2717 & 0.049 & 44 & 0.95 & 1.20 & 1.28 & 1.47 & 0.269 \\
\hline A 2734 & 0.061 & 82 & 1.00 & 1.46 & 1.89 & 1.50 & 0.211 \\
\hline A 3128 & 0.060 & 236 & 0.88 & 1.89 & 1.46 & 1.71 & 0.000 \\
\hline A 3158 & 0.059 & 209 & 1.00 & 2.25 & 1.82 & 1.71 & 0.650 \\
\hline A3266 & 0.059 & 297 & 1.00 & 2.99 & 2.46 & 1.67 & 0.086 \\
\hline A3376 & 0.046 & 102 & 1.00 & 1.72 & 1.79 & 1.66 & 0.624 \\
\hline A3395 & 0.050 & 159 & 0.96 & 1.64 & 1.90 & 1.68 & 0.031 \\
\hline A3490 & 0.069 & 87 & 0.95 & 1.67 & - & 1.67 & 0.721 \\
\hline A3497 & 0.068 & 102 & 0.90 & 1.59 & - & 1.74 & 0.149 \\
\hline A3528a & 0.054 & 68 & 1.00 & 2.12 & 1.81 & 1.51 & 0.404 \\
\hline A $3528 b$ & 0.054 & 70 & 0.97 & 1.88 & 1.84 & 1.55 & 0.362 \\
\hline A3530 & 0.054 & 58 & 0.95 & 1.26 & 1.70 & 1.56 & 0.318 \\
\hline A 3532 & 0.055 & 61 & 0.97 & 1.21 & 1.80 & 1.52 & 0.527 \\
\hline A3556 & 0.048 & 139 & 0.99 & 1.24 & 1.40 & 1.70 & 0.321 \\
\hline A3558 & 0.047 & 164 & 1.00 & 2.00 & 1.87 & 1.76 & 0.233 \\
\hline A3560 & 0.049 & 133 & 1.00 & 1.74 & 1.65 & 1.74 & 0.148 \\
\hline A3667 & 0.055 & 84 & 1.00 & 2.16 & 2.12 & 1.41 & 0.517 \\
\hline A3716 & 0.045 & 88 & 0.97 & 1.72 & - & 1.48 & 0.127 \\
\hline A3809 & 0.063 & 117 & 0.72 & 1.25 & - & 1.67 & 0.510 \\
\hline A3880 & 0.058 & 75 & 0.99 & 1.60 & 1.23 & 1.41 & 0.795 \\
\hline A4059 & 0.049 & 84 & 1.00 & 1.68 & 1.74 & 1.56 & 0.106 \\
\hline IIZW108 & 0.048 & 48 & 0.98 & 1.10 & - & 1.46 & 0.138 \\
\hline MKW3s & 0.045 & 61 & 1.00 & 1.28 & 1.67 & 1.55 & 0.978 \\
\hline RX0058 & 0.048 & 27 & 0.96 & 1.30 & 0.89 & 1.37 & 0.562 \\
\hline RX1022 & 0.054 & 55 & 1.00 & 1.51 & 1.07 & 1.29 & 0.201 \\
\hline $\mathrm{RX} 1740$ & 0.044 & 31 & 1.00 & 1.21 & - & 1.40 & 0.691 \\
\hline Z1261 & 0.065 & 13 & 0.85 & 1.14 & - & 1.14 & 0.949 \\
\hline Z2844 & 0.050 & 61 & 1.00 & 1.15 & - & 1.56 & 0.145 \\
\hline Z8338 & 0.049 & 74 & 0.95 & 1.54 & - & 1.59 & 0.574 \\
\hline Z8852 & 0.041 & 74 & 1.00 & 1.61 & 1.35 & 1.63 & 0.111 \\
\hline
\end{tabular}

Notes. $N_{\mathrm{m}}$ is the number of members and $f_{\mathrm{m}, \text { vir }}$ is the fraction of members at distances $\leq r_{200, \sigma}$ from the BCG centers.

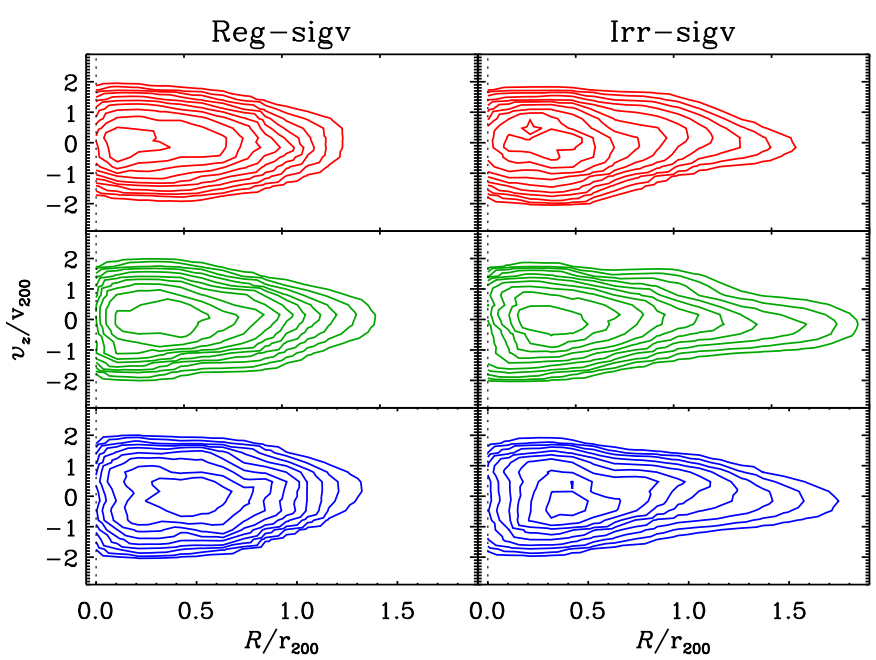

Fig. 3. Adaptive-kernel density maps in projected phase-space (restframe velocities versus distances from the cluster centers) for the Regsigv (left panels) and Irr-sigv (right panels) stacks. Radii and velocities are in normalized units, using $r_{200, \sigma}$ and $v_{200, \sigma}$ for the normalization of radii and velocities, respectively. Upper panel: E, middle panel: S0, lower panel: S. Density contours are space logarithmically.

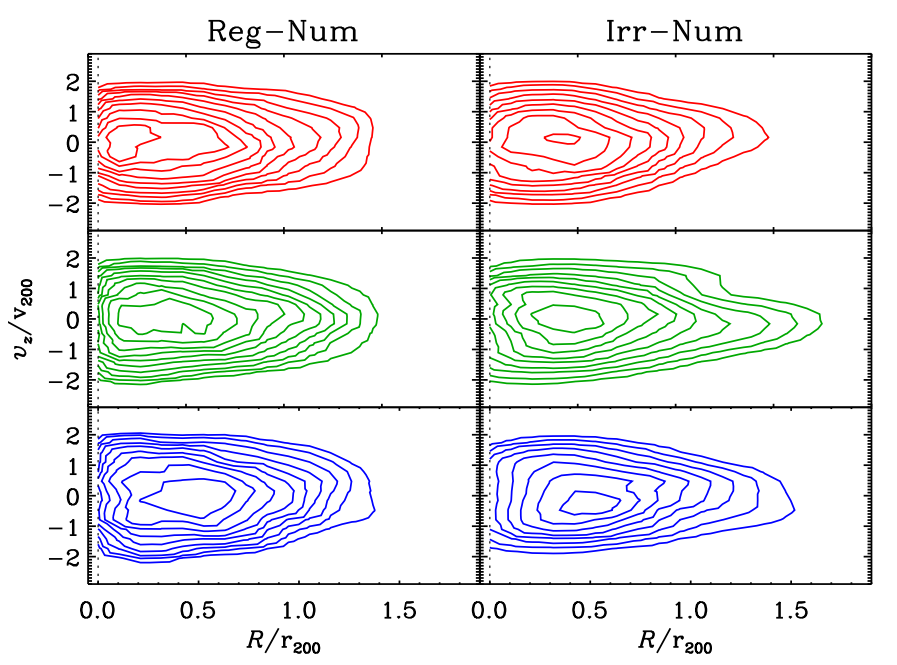

Fig. 4. Same as Fig. 3, but for the Reg-Num (left panel) and Irr-Num (right panel) samples, and using $r_{200, N}, v_{200, N}$ in lieu of $r_{200, \sigma}, v_{200, \sigma}$.

$\geq 0.1$ ). This is also true of the Irr stacks obtained using the temp and Num scalings. On the other hand, the Irr stack obtained using the sigv scaling is significantly different from the other two Irr stacks (probability $\leq 0.001$ ). For this reason, we consider in the following both the stacks obtained using the sigv scaling, that we name Reg-sigv and Irr-sigv, and the stacks obtained using the Num scaling, that we name Reg-Num and Irr-Num (we prefer the latter to the stacks obtained using the temp scaling because of better statistics).

We show the PPSD of the Reg-sigv and Irr-sigv stacks in Fig. 3, and those of the Reg-Num and Irr-Num stacks in Fig. 4, separately for E, S0, and S members. The 2D K-S test indicates that $\mathrm{E}, \mathrm{S} 0$, and $\mathrm{S}$ galaxies have significantly (probability $<0.01$ ) different PPSDs in all four stacks, even when restricting the comparison to the radial range covered by both the populations being compared. 


\section{The surface number density profiles}

To obtain the number density profiles, $\Sigma(R)$, of our clusters we use the photometric data rather than the spectroscopic data to avoid the need for the correction for incompleteness of the spectroscopic sample. We consider all galaxies in the cluster fields down to the magnitude limit $V=19$, to ensure that we are using similar populations of cluster galaxies for the determination of $\Sigma(R)$ and VDP, since most of our spectroscopic cluster members (that we use for the determination of the VDP - see Sect. 5) are brighter than $V=19$ (see Fig. 1). In determining the $\Sigma(R)$ we correct the observed galaxy counts by the fractions of the circular annuli covered by the imaging observations.

We consider two models for the fit of $\Sigma(R)$ :

1. the NFW model by Navarro et al. (1996), whose surface density profile was analytically derived by Bartelmann (1996), as well as by Łokas \& Mamon (2001) in a slightly simpler form;

2. the King (1962) model, $\Sigma(R) \propto\left[1+\left(R / r_{\mathrm{c}}\right)^{2}\right]^{-1}$.

We add a constant surface density of background galaxies $\left(\Sigma_{\mathrm{bg}}\right)$ to both models. The NFW profile is theoretically motivated and has been shown to fit the $\Sigma(R)$ of cluster galaxies satisfactorily well (see, e.g., Biviano \& Girardi 2003; Lin et al. 2004). The King model differs from NFW in that it is characterized by a central core and a sharper transition from the inner 3D density slope (zero for King, -1 for NFW) to the outer slope of -3 .

We use a maximum likelihood technique applied to the distribution of projected radii to find the best-fit parameters of the two models, namely the scale radius ( $r_{\mathrm{s}}$ in the case of the NFW model, and $r_{\mathrm{c}}$ in the case of the King model), and the constant background surface density, $\Sigma_{\mathrm{bg}}$, in units of the normalization of the model. The normalization of the model is not a free parameter, as it is constrained by the condition that the integral of the surface number density over the considered area is equal to the number of observed galaxies (down to the chosen magnitude limit). We prefer to leave $\Sigma_{\text {bg }}$ a free parameter in the fit, rather than using an average background density for all clusters, to account for cosmic variance.

We limit the fit to the region between $50 \mathrm{kpc}$ (basically excluding the $\mathrm{BCG}$ ) and $R_{\text {lim }}$, the radius of the most distant galaxy from the BCG where the fraction of the area covered by the imaging observations is $>0.6$. For most clusters $R_{\text {lim }} \geq 1 \mathrm{Mpc}$, except for A168, where $R_{\text {lim }}<0.5 \mathrm{Mpc}$. For this reason, we exclude A168 from the following analysis, and consider only the remaining 67 clusters from the $N_{\mathrm{m}} \geq 30$ sample.

After fitting a model of $\Sigma(R)$ to the projected radii of all the galaxies, we then fit separately the corresponding profiles of E, S0, and S galaxies. In this second step of our procedure, we use a single-parameter fit, and adopt the best-fit $\Sigma_{\text {bg }}$ value obtained by fitting $\Sigma(R)$ to the full galaxy set (hereafter, the "All" sample), multiplied by the relative fractions of E, S0, and $\mathrm{S}$ galaxies in the field. We take these fractions from Figs. 7 and 9 of Bamford et al. (2009), 0.1, 0.2, and 0.7 for E, S0, and S galaxies, respectively. These values are based on the data set of $M_{B} \leq-18.3$ galaxies of Postman \& Geller (1984, after conversion to our adopted cosmology), Given typical $B-V$ galaxy colors (e.g., Fukugita et al. 1995), and the average redshift of galaxies in our sample, $M_{B}=-18.3$ corresponds to an apparent magnitude limit $V \sim 18$, and a stellar mass limit $\log M_{\star} \sim 9.5$ (using the relations of Bell \& de Jong 2001). The sample we use for the $\Sigma(R)$ determination is $\sim 1$ mag fainter that of Postman \& Geller (1984), corresponding to a stellar mass $\log M_{\star} \sim 9.1$. The field morphological fractions change significantly with stellar mass, but not below $\log M_{\star} \sim 9.5$, so the morphological fractions of Bamford et al. (2009) are applicable to our sample.

The statistics are good enough to allow fitting 47/42/33 of the 67 individual cluster E/S0/S surface number density profiles. Using the Bayesian information criterion

$\mathrm{BIC}=-2 \ln \mathcal{L}_{\max }+\left(\ln N_{\mathrm{d}}\right) N_{\mathrm{p}}$,

where $N_{\mathrm{d}}, N_{\mathrm{p}}$ are the number of data and free parameters, respectively, we establish that the projected NFW model provides a better fit than the King model to 84, 78, and 67\% (respectively 80,63 , and $56 \%$ ) of the E, S0, and S galaxy surface density profiles for the Reg (respectively Irr) cluster set. In general, the spatial distribution of galaxies is therefore better described by the NFW model than by the King model, but this is less commonly the case for galaxies of later types and in irregular clusters.

To define the model profile to be used for the stacks, we then average the values of the scale radius parameter of each cluster, in units of the cluster $r_{200}\left(r_{200, \sigma}\right.$ or $\left.r_{200, N}\right)$, using the number of cluster members as weights. While these values are obtained using a subset of all the clusters that contribute to the stack (i.e., those with sufficient statistics for allowing the $\Sigma(R)$ determination and fit), we can assume that each average is representative of the full sample. We expect this assumption not to introduce any significant bias in our analysis, given that clusters with poor statistics (few member galaxies) do not contribute much to the stacks anyway.

In Figs. 5 and 6 we compare the average best-fit (projected) NFW and King models to the binned $\Sigma(R)$, obtained from the four spectroscopic stack samples Reg-sigv, Irr-sigv, Reg-Num, and Irr-Num. In estimating the stack binned $\Sigma(R)$ we take into account two completeness corrections, namely we correct for the incompleteness of the spectroscopic sample, and for the fact that, in the outer regions, at a given radius only a subset of clusters contribute to the stack. This second correction is described in Merrifield \& Kent (1989), Biviano et al. (2002). The normalization of the $\Sigma(R)$ model fits are adjusted to fit the binned $\Sigma(R)$. Overall, the visual impression is that of a good agreement between the binned and fitted $\Sigma(R)$, considering that they have been obtained by very different procedures and that the completeness corrections for the spectroscopic samples are very uncertain at large radii.

To estimate the effect of adopting the BCG position as a cluster center, we repeated our analysis by adopting an alternative center definition, namely the peak of the cluster X-ray emission. The average distance of the X-ray and BCG centers for our clusters is $\sim 100 \mathrm{kpc}$. This difference does not result in a significant difference of the PPSDs of the stacked samples, according to a 2D Kolmogorov-Smirnov test, for any of the morphological classes and adopted scalings. Changing the center definition has also a negligible effect on the membership determination for the different stacks. A more quantitative comparison is provided by Fig. 7, where we show the distribution of the ratios of the best-fit scale radii of our clusters, obtained using the two center definitions, for the three morphological classes and both the NFW and King models. Most of the ratios are within 0.03 of unity. As a result, the effect of a different center choice on the stacked NDPs is even smaller. We also checked that the different center choice has little effect on the VDPs (described in Sect. 5).

The results of the $\Sigma(R)$ fits are listed in Table 2, where we give the values of the average NFW and King scale radii in units of $\left\langle r_{200}\right\rangle$, as previously explained. We find that independent of the model, the scale radius increases from E to S0 to $\mathrm{S}$ (see Table 2 and Fig. 8), as expected from the well-known 


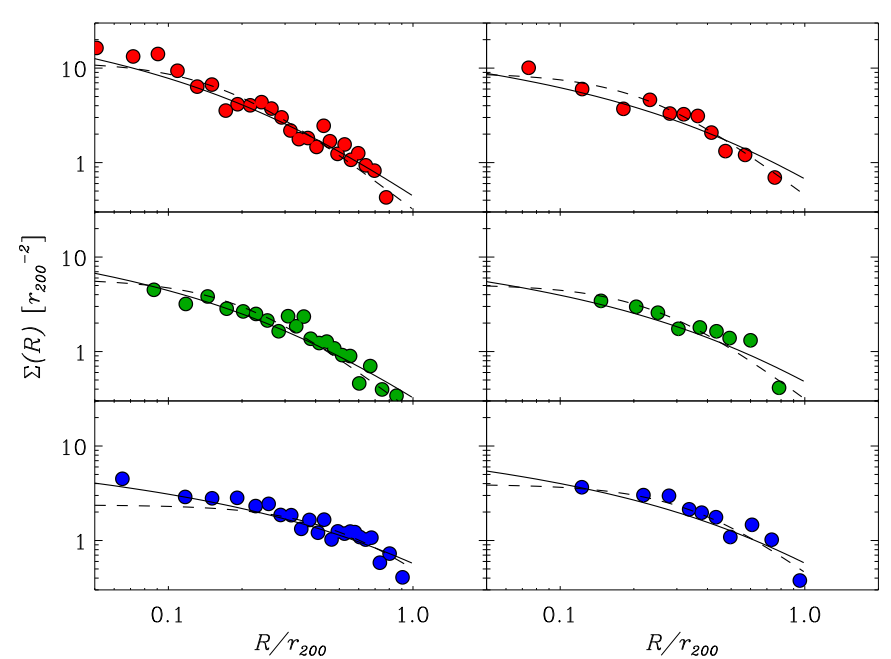

Fig. 5. Projected surface number density profiles for E (top panel), S0 (middle panel), and S (bottom panel) galaxies, in the Reg-sigv (lefthand panels) and Irr-sigv (right-hand panels) stacks, as determined using the spectroscopic samples of cluster members, and the completeness weights. Radii are in units of $r_{200, \sigma}, \Sigma(R)$ are in units of $r_{200, \sigma}^{2}$. The solid (respectively dashed) curves are the best-fit (projected) NFW (respectively King) profiles, as obtained by averaging the results of maximum likelihood fits to the $\Sigma(R)$ of individual clusters, obtained using the photometric sample. Poisson error bars are smaller than the size of the symbols, but do not include the completeness uncertainties.

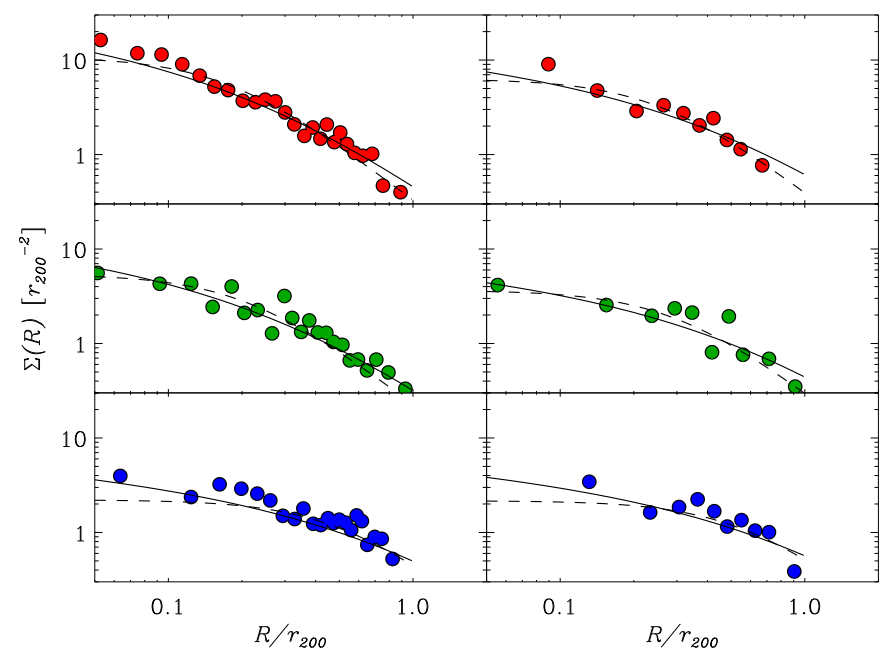

Fig. 6. Same as Fig. 5, but for the Reg-Num and Irr-Num samples, using $r_{200, N}$ in lieu of $r_{200, \sigma}$.

morphology-density relation (Dressler 1980). This is true both for Reg and Irr clusters, and for both $r_{200}$ scalings.

We also find that the concentrations, $r_{200} / r_{\mathrm{s}}$ and $r_{200} / r_{\mathrm{c}}$ for the NFW and King models, respectively, of the spatial distributions of E and S0 galaxies are higher in Reg than in Irr clusters, again independently of the $r_{200}$ scaling. This is not true for the concentration of the spatial distribution of $\mathrm{S}$ galaxies. We provide a possible interpretation for this new result. Dynamically relaxed cluster-size halos from cosmological simulations are known to display a higher concentration (per given mass) than their unrelaxed counterparts (e.g., Jing 2000; Neto et al. 2007). This is probably the consequence of recent (major) mergers occurring in unrelaxed clusters. This could also explain the

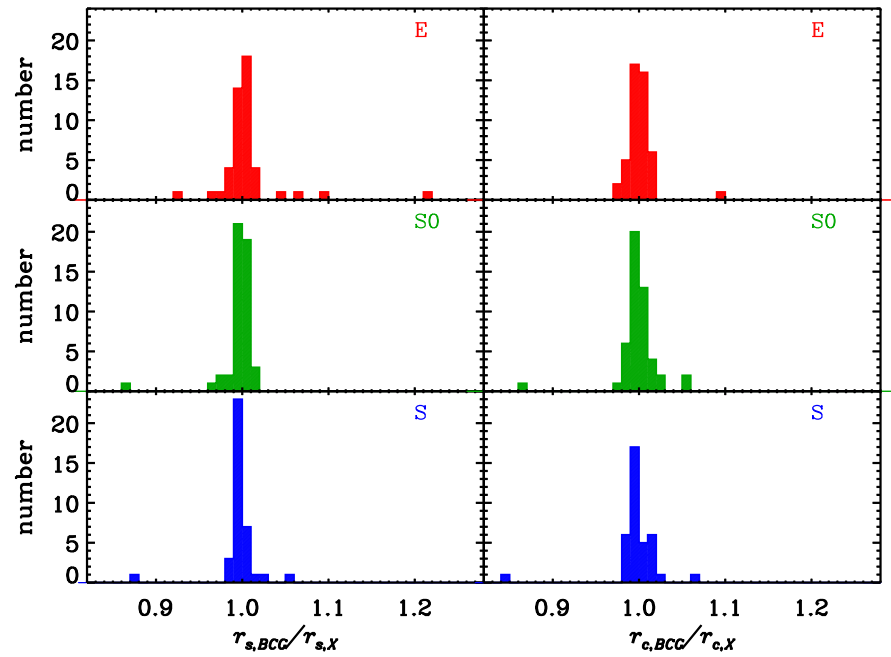

Fig. 7. Histogram distributions of the ratio of best-fit NFW (left panels) and King (right panels) model scale radius parameters for individual clusters, using BCG and X-ray centers, for the E, S0, and S populations in the top, middle, and bottom panels, respectively.

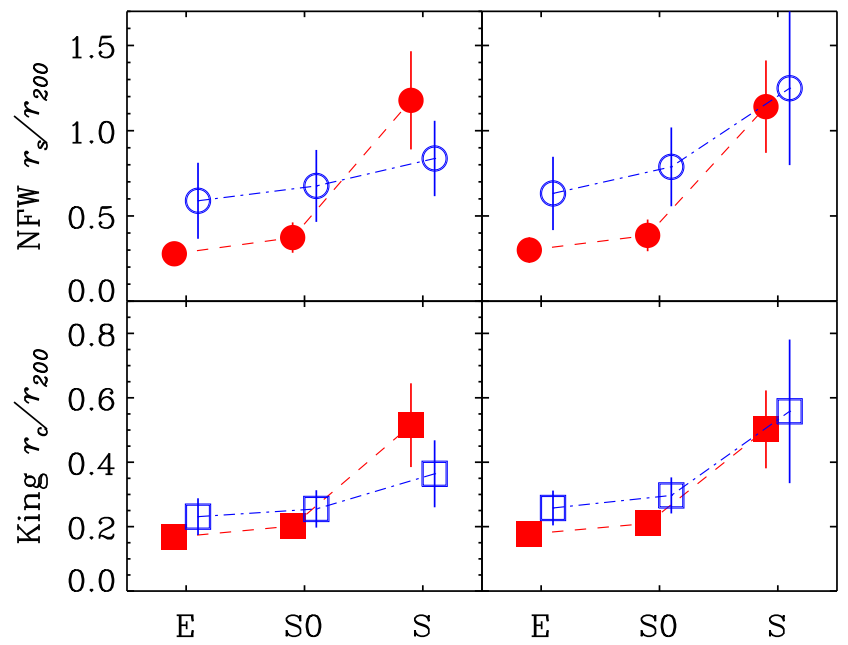

Fig. 8. Normalized scale radius as a function of the galaxy population for the Reg (red dashed lines and filled symbols) and Irr (blue dashdotted lines and open symbols) sample. Top panels and dots (respectively bottom panels and squares) are for the NFW (respectively King) scale-radius. Left (respectively right) panels are for the $r_{200, \sigma}$ (respectively $r_{200, N}$ ) scaling. Error bars are computed according to Eq. (16) of Beers et al. (1990).

higher concentration of E and S0 galaxy distributions in Reg clusters, if these kind of galaxies are good tracers of the mass distribution. On the other hand, the insensitivity of the S spatial distribution to their cluster relaxation state suggests that $S$ galaxies are recent newcomers in the cluster potential, and that they might not have settled down in a dynamical equilibrium configuration yet.

\section{The line-of-sight velocity dispersion profiles}

We determine the line-of-sight velocity dispersion profiles (VDPs in the following) of different galaxy populations in our four stack samples, by computing the biweight (Beers et al. 1990) velocity dispersion in concentric radial bins. We need not worry about completeness in this analysis. In fact, observational selection is unlikely to operate in velocity space within the 


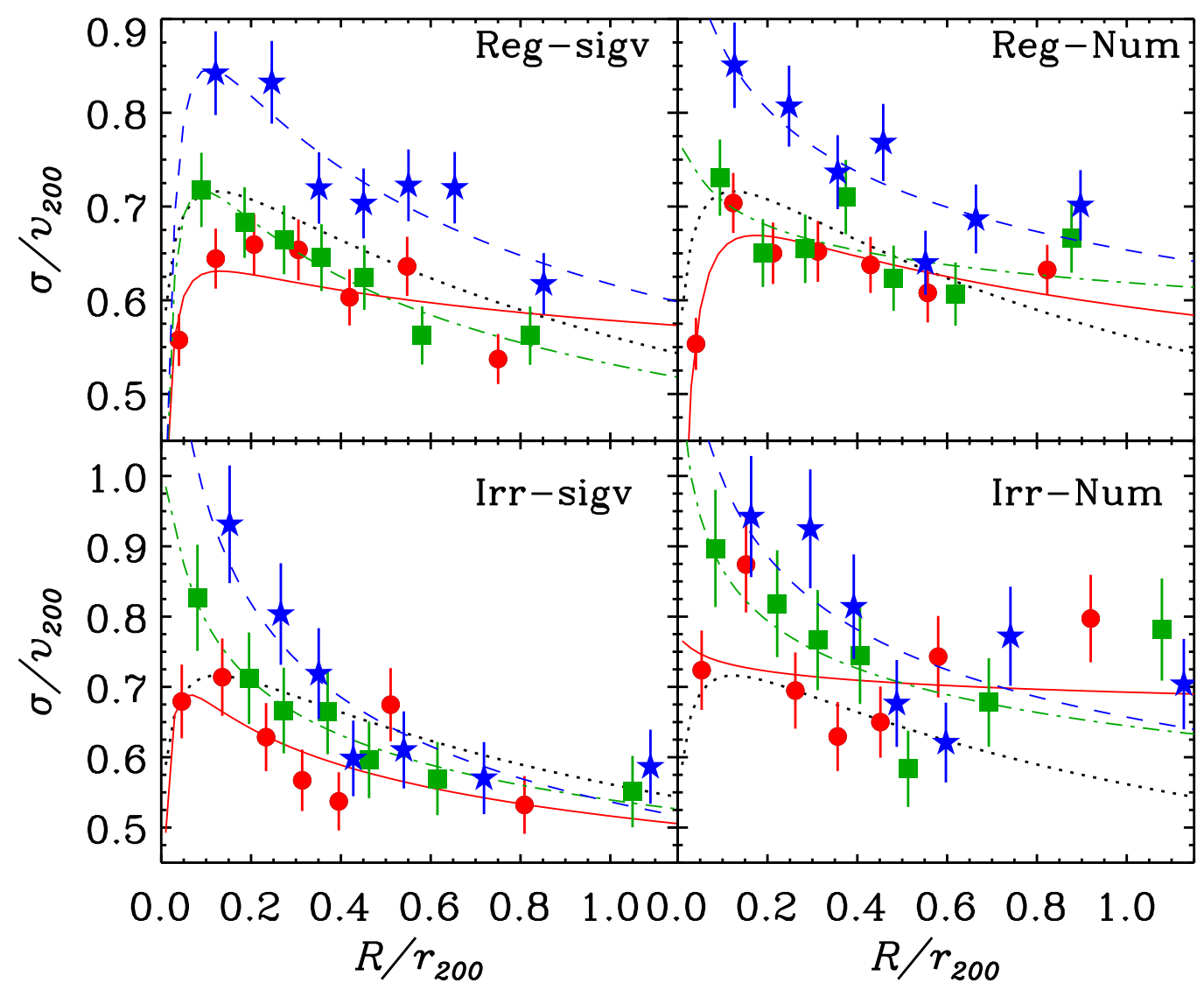

Fig. 9. Line-of-sight velocity dispersion profiles of different cluster galaxy populations in the four stack samples (top-left: Reg-sigv; top-right: Reg-Num; bottom-left: Irr-sigv; bottom-right: Irr-Num). Red dots, green squares, and blue stars represent the VDPs of E, S0, and S galaxies, respectively. The red solid, green dash-dotted, and blue dashed lines represent model fits described by Eq. (2) and Table 3. The black dotted line represents the theoretical VDP predicted for a cluster with a NFW mass distribution of concentration $c \equiv r_{200} / r_{\mathrm{s}}=3$ and with a velocity anisotropy profile for its galaxies given by Eq. (60) in Mamon \& Łokas (2005) with $r_{\mathrm{a}}=r_{\mathrm{s}}$. Error bars are computed according to Eq. (16) of Beers et al. (1990).

Table 2. Surface number density profiles of cluster galaxy populations.

\begin{tabular}{llrrr}
\hline \hline Stack & Class & NFW $\left\langle r_{\mathrm{s}} / r_{200}\right\rangle$ & King $\left\langle r_{\mathrm{c}} / r_{200}\right\rangle$ & $\left\langle r_{200}\right\rangle^{a}$ \\
\hline Reg-sigv & E & $0.278 \pm 0.065$ & $0.167 \pm 0.025$ & $1.749 \pm 0.064$ \\
& S0 & $0.373 \pm 0.089$ & $0.202 \pm 0.027$ & \\
& S & $1.178 \pm 0.288$ & $0.515 \pm 0.130$ & \\
Irr-sigv & E & $0.589 \pm 0.223$ & $0.231 \pm 0.057$ & $1.976 \pm 0.157$ \\
& S0 & $0.676 \pm 0.211$ & $0.255 \pm 0.058$ & \\
& S & $0.837 \pm 0.221$ & $0.364 \pm 0.104$ & \\
Reg-Num & E & $0.300 \pm 0.075$ & $0.177 \pm 0.027$ & $1.629 \pm 0.022$ \\
& S0 & $0.386 \pm 0.093$ & $0.210 \pm 0.027$ & \\
Irr-Num & S & $1.141 \pm 0.271$ & $0.502 \pm 0.121$ & \\
& E & $0.632 \pm 0.215$ & $0.258 \pm 0.054$ & $1.652 \pm 0.053$ \\
& S0 & $0.788 \pm 0.231$ & $0.297 \pm 0.056$ & \\
& S & $1.249 \pm 0.451$ & $0.558 \pm 0.223$ & \\
\hline
\end{tabular}

Notes. ${ }^{(a)}\left\langle r_{200}\right\rangle$, in Mpc, is the weighted average of the individual cluster $r_{200}$ estimates, namely $r_{200, \sigma}$ for the Reg-sigv and Irr-sigv stacks, and $r_{200, N}$ for the Reg-Num and Irr-Num stacks.

(relatively) narrow velocity range spanned by cluster members. So, even if some radial bins lack a higher fraction of galaxies than others, the lacking galaxies are unlikely to occupy a preferred location in the global velocity distribution of cluster members, and therefore the velocity dispersion estimate remains unaffected by the sample incompleteness. To confirm this expectation we have performed 100 bootstrap simulations, randomly extracting from each radial bin a random number of galaxies from the observed sample. The relative incompleteness across different radial bins is at most a factor 2 . Therefore we choose to simulate the effect of such a random sampling by selecting different fractions of observed galaxies in the different bins, where these fractions are randomly chosen uniformly sampling the range of 50-100\% completeness. We confirm that introducing different levels of radial completeness in the galaxy distribution does not introduce any significant bias in the VDP determination. We therefore use the full spectroscopic sample for this analysis.

The VDPs are shown in Fig. 9 separately for the E, S0, and S populations in the four stacks. We used seven bins for each subsample, with the same number of galaxies per bin. The dotted line in each panel of the figure is the predicted VDP ("theoretical VDP" in the following) for a cluster with a NFW mass distribution of concentration $c \equiv r_{200} / r_{\mathrm{s}}=3$, typical of massive clusters (e.g., Groener et al. 2016), and with a velocity anisotropy profile of cluster galaxies given by Eq. (60) in Mamon \& Łokas (2005) with anisotropy radius equal to the NFW mass profile scale radius, $r_{a}=r_{\mathrm{s}}$ (this anisotropy profile fits cluster-size simulated halos quite well, Mamon et al. 2010).

In Fig. 9 we also show best-fit models to the binned VDPs of E, S0, and S galaxies. We use the following model:

$\frac{\sigma_{\mathrm{los}}}{v_{200}}=s_{0} \frac{X}{a_{\sigma}}\left(1+\frac{X}{a_{\sigma}}\right)^{-\eta}$, 
A. Cava et al.: Structural and dynamical modeling of WINGS clusters. I.

Table 3. Best-fit parameters of model VDPs.

\begin{tabular}{llrrcr}
\hline \hline Stack & Class & $\begin{array}{r}s_{0} \\
{\left[v_{200}\right]}\end{array}$ & $\begin{array}{r}a_{\sigma} \\
{\left[10^{-3} r_{200}\right]}\end{array}$ & $\eta$ & $\chi^{2}$ \\
\hline Reg-sigv & E & 1.17 & 30.5 & 1.21 & 3.7 \\
& S0 & 1.20 & 8.4 & 1.16 & 1.8 \\
& S & 1.59 & 27.2 & 1.25 & 4.1 \\
Irr-sigv & E & 1.10 & 10.0 & 1.16 & 7.4 \\
& S0 & 1.69 & 2.0 & 1.19 & 2.8 \\
& S & 2.40 & 4.5 & 1.28 & 4.4 \\
Reg-Num & E & 1.02 & 23.8 & 1.14 & 2.9 \\
& S0 & 1.08 & 0.7 & 1.08 & 3.9 \\
& S & 1.46 & 2.8 & 1.14 & 6.4 \\
Irr-Num & E & 0.87 & 0.2 & 1.03 & 12.3 \\
& S0 & 1.75 & 1.5 & 1.16 & 21.0 \\
& S & 1.99 & 3.6 & 1.20 & 8.6 \\
\hline
\end{tabular}

Notes. These are the parameters of the model given by Eq. (2). The $\chi^{2}$ values are obtained for the fits to seven data-points in each case.

where $X=R / r_{200}$, and $s_{0}$ and $a_{\sigma}$ are in units of $v_{200}$ and $r_{200}$, respectively. We find this model to provide an adequate fit to (almost) all our VDPs. In Table 3 , we provide the $\chi^{2}$ values of the fits, together with the best-fit parameters. Since this model is a useful parametrization of the cluster VDPs, but has no physical meaning, we consider it not useful to estimate (and list) the uncertainties on the fitting parameters.

The comparison of the observed VDPs with the theoretical VDP (dotted line in Fig. 9) suggests that our stack clusters should have an internal structure that is not too dissimilar from the one assumed to derive the theoretical VDP, at least for a certain class of galaxies. Perhaps the stack with the VDP that most strongly deviates from the theoretical VDP is Irr-Num. We postpone a full dynamical analysis of our data set to Paper II in this series (Mamon et al., in prep.).

What is more pertinent to this paper is the direct comparison of the VDPs of the different classes. To perform this comparison, we re-determine the velocity dispersion of each galaxy population within fixed radial bins, common to all classes (and all stacks). Specifically, we use seven radial bins with inner and outer radii $(i-1) \times 0.15 r_{200}$ and $i \times 0.15 r_{200}$, for $i=1,7$. We then evaluate the $\chi^{2}$ statistic given by

$\chi^{2}=\sum_{i=1}^{7} \frac{\left(\sigma_{x, i}-\sigma_{y, i}\right)^{2}}{\delta_{x, i}^{2}+\delta_{y, i}^{2}}$

where $\sigma_{x, i}, \sigma_{y, i}$ are the velocity dispersions of populations $x$ and $y$ in the $i$ th radial bin, and $\delta_{x, i}, \delta_{y, i}$ are their errors. We compare this statistic to a $\chi^{2}$ distribution to obtain the probabilities that the two populations have the same VDPs. These are listed in Table 4.

We see that E and S0 galaxies have similar VDPs in all stacks, although the S0 VDP tends to be steeper than the E VDP. On the other hand, E and S galaxies have different VDPs in all stacks, and the S VDP is above the E VDP, especially near the center. In the outer regions, the S VDP becomes more similar to the E VDP because it is steeper. Our results are in agreement with the findings of Adami et al. (1998, see their Fig. 9) and consistent with the results of Biviano \& Katgert (2004, see their Fig. 2), although the latter did not distinguish E from S0.
Table 4. Comparison of the cluster galaxy population VDPs.

\begin{tabular}{llrrr}
\hline \hline \multirow{2}{*}{ Stack } & Classes & Prob & \multicolumn{2}{c}{ VDP ratio $y / x$} \\
& $x$ vs. $y$ & & $\leq 0.5 r_{200}$ & $>0.5 r_{200}$ \\
\hline Reg-sigv & E vs. S0 & 0.184 & $1.08 \pm 0.08$ & $1.04 \pm 0.09$ \\
& E vs. S & $<\mathbf{0 . 0 0 1}$ & $\mathbf{1 . 2 7} \pm \mathbf{0 . 1 1}$ & $\mathbf{1 . 2 2} \pm \mathbf{0 . 0 6}$ \\
& S0 vs. S & $<\mathbf{0 . 0 0 1}$ & $\mathbf{1 . 1 7} \pm \mathbf{0 . 0 4}$ & $\mathbf{1 . 1 7} \pm \mathbf{0 . 0 4}$ \\
Irr-sigv & E vs. S0 & 0.125 & $1.15 \pm 0.07$ & $0.98 \pm 0.10$ \\
& E vs. S & $\mathbf{0 . 0 1 0}$ & $\mathbf{1 . 3 5} \pm \mathbf{0 . 0 7}$ & $\mathbf{1 . 0 3} \pm \mathbf{0 . 0 4}$ \\
& S0 vs. S & 0.194 & $1.08 \pm 0.10$ & $1.06 \pm 0.11$ \\
Reg-Num & E vs. S0 & 0.287 & $1.04 \pm 0.08$ & $0.96 \pm 0.04$ \\
& E vs. S & $<\mathbf{0 . 0 0 1}$ & $\mathbf{1 . 2 4} \pm \mathbf{0 . 0 6}$ & $\mathbf{1 . 1 2} \pm \mathbf{0 . 0 2}$ \\
& S0 vs. S & $\mathbf{0 . 0 0 8}$ & $\mathbf{1 . 1 1} \pm \mathbf{0 . 0 0}$ & $\mathbf{1 . 0 9} \pm \mathbf{0 . 0 4}$ \\
Irr-Num & E vs. S0 & 0.084 & $1.15 \pm 0.11$ & $0.94 \pm 0.09$ \\
& E vs. S & $\mathbf{0 . 0 1 7}$ & $\mathbf{1 . 2 6} \pm \mathbf{0 . 0 5}$ & $\mathbf{0 . 9 4} \pm \mathbf{0 . 0 2}$ \\
& S0 vs. S & 0.150 & $1.12 \pm 0.07$ & $0.91 \pm 0.15$ \\
\hline
\end{tabular}

Notes. "Prob" indicates the probability that the VDPs of the $x$ and $y$ populations are the same. We consider probabilities $<0.05$ as indicating a significant difference in the VDPs. These cases are highlighted in boldface in the table. The VDP ratios are computed in two radial bins.

However, in addition to these previous studies, here we distinguish regular from irregular clusters.

An entirely new result of our analysis is how the S0 galaxies' VDP compares to the VDPs of the other two classes in Reg and Irr clusters. In Reg clusters, the S0 VDP is significantly different from the $S$ VDP, being systematically below at all radii. In Irr clusters, the S0 VDP remains below the S VDP at all radii, but the difference is no longer significant. It therefore appears that while S0 galaxies in Reg clusters have similar velocity distributions to E galaxies, in Irr clusters, the velocity distribution of S0 galaxies is intermediate between that of $\mathrm{S}$ and $\mathrm{E}$.

The different behavior of the S0 VDP with respect to the other classes' VDPs in Reg and Irr clusters suggests that S0 galaxies in Irr clusters are a younger population than S0 galaxies in Reg clusters. In Irr clusters, relatively to Reg clusters, a larger fraction of S0 galaxies is likely to have relatively recently evolved from infalling $\mathrm{S}$ galaxies.

\section{Discussion}

In Sect. 3 we have shown that the PPSDs (for all the considered stacks and scalings) are statistically different for the three classes of galaxies considered in this study: E, S0, and S. This result is at variance with that of Biviano et al. (2002), based on the European Southern Observatory Nearby Abell Cluster Survey (ENACS; Katgert et al. 1996, 1998), where no statistical evidence was found for different PPSDs of E and S0. We argue that this might be due to Biviano et al. (2002) using the mixed morphological and spectroscopical classification of galaxies by Thomas \& Katgert (2006b), which differs from the pure morphological classification used in this work. It is plausible that a substantial fraction of spectroscopically-classified S0 galaxies are in fact morphological E galaxies (Thomas \& Katgert 2006b), and this contamination would reduce the detectability of any intrinsic difference in the distributions of the two populations.

In Sects. 4 and 5 we have dissected the PPSDs into their spatial and velocity components. The spatial distributions were parametrized by the scale- or core-radius of the best-fitting NFW or King models to the surface number density profiles of cluster galaxies. We find that in general the NFW profile 
is preferred over the cored-King profile, but this is less and less evident for late-type galaxies and Irr clusters. The spatial distributions become increasingly more concentrated towards earlier morphological classes, and this reflects the wellknown morphology-radius relation (Sanromà \& Salvador-Solé 1990; Whitmore et al. 1993; Fasano et al. 2015, and references therein). The velocity distributions were described in terms of the velocity dispersion profiles, that we modeled with the analytical profile of Eq. (3). The velocity dispersion profile has a higher normalization and is steeper for later morphological classes, confirming previous results (Adami et al. 1998; Biviano \& Katgert 2004).

These morphological segregations in the spatial and velocity distributions have been generally interpreted as $\mathrm{E}$ being the oldest cluster population, followed by S0, and then S (e.g., Dressler et al. 1997; Biviano \& Katgert 2003; Biviano 2011, and references therein). The high- $z$ cluster environment is believed to have favored the morphological evolution of field galaxies into $\mathrm{E}$, presumably via mergers, before the cluster velocity dispersion grew too high for mergers to be effective. Later evolution concerns the transformation of field S into cluster S0, and there is direct evidence that the fraction of cluster $\mathrm{S}$ decreases with time since $z \sim 1$, at the same time as the fraction of cluster S0 increases (Postman et al. 2005; Smith et al. 2005; Desai et al. 2007).

We have found that the distributions of E, S0, and S galaxies depend on the kind of clusters they belong to. In particular, both $\mathrm{E}$ and $\mathrm{S} 0$ have more concentrated spatial distributions (i.e., greater $r_{200} / r_{\mathrm{S}}$ or $r_{200} / r_{\mathrm{c}}$ ) in Reg than in Irr clusters. This is not the case for $\mathrm{S}$.

Dynamically relaxed cluster-size halos from cosmological simulations have higher mass density concentrations than unrelaxed halos of the same mass (e.g., Jing 2000; Neto et al. 2007). The fact that we see the same trend observationally in the distribution of E and S0, but not S, suggests that E and S0 galaxies are good tracers of the cluster mass distribution. $S$ galaxies are not, probably because they are a recently accreted population in both Reg and Irr clusters, while (most) E and S0 galaxies have resided in the cluster environment for a sufficiently long time to settle down into a spatial distribution similar to that of the whole cluster mass.

Another important difference between Reg and Irr clusters is evidenced in the VDP of S0. This appears very similar to the VDP of E galaxies in Reg clusters, and intermediate between those of $\mathrm{E}$ and $\mathrm{S}$ in Irr clusters. S0 galaxies share the velocity distribution of $\mathrm{E}$ in the more evolved Reg clusters, presumably because they have had the time to settle down in the cluster potential, unlike in the less evolved Irr clusters. The average time since morphological evolution of S0 galaxies from infalling $\mathrm{S}$ galaxies must be shorter in Irr than in Reg clusters. It is possible that this difference in age could be visible in the S0 spectra, but such an analysis is beyond the scope of this work.

The different spatial and velocity distributions of S0 and $S$ galaxies suggest that it is the cluster environment that operates the transformation process between these two morphological types. In fact, if most $\mathrm{S} 0$ galaxies evolve from $\mathrm{S}$ galaxies in groups before being accreted onto clusters (the so-called "preprocessing"; Zabludoff \& Mulchaey 1998; Balogh et al. 1999; Mahajan 2013), S and S0 should share similar spatial and velocity distributions. S0 galaxies are therefore expected to be the result of a transformation process of $S$ galaxies operated by the cluster environment. The different PPSD of S0 and S galaxies might result from the fact that the morphological evolutionary processes are more effective in the central cluster regions (e.g., Moran et al. 2007). In particular, ram-pressure stripping (Gunn \& Gott 1972), and/or starbursts induced by tidal compression by the cluster gravitational field, especially while the galaxies are accreted in groups (the so-called "post-processing"; Bekki 1999; Oemler et al. 2009; Vijayaraghavan \& Ricker 2013; Stroe et al. 2015; Jaffé et al. 2016), likely play a relevant role. This compression could drive the rapid consumption of gas funneled into the central galaxy region and the build-up of a central bulge.

Previous works have indicated that $\geq 2 / 3$ of cluster $\mathrm{S} 0$ form after $z \sim 0.4$, that is, in the last 3-4 Gyr (Postman et al. 2005; Smith et al. 2005; Desai et al. 2007). This sets an upper limit to the timescale for morphological evolution. We also observe that SO and S have different PPSDs, so also this differentiation must occur on a timescale $\lesssim 3$ Gyr. This upper limit is consistent with the dynamical timescale, $t_{\mathrm{dyn}} \sim(G\langle\rho\rangle)^{-1 / 2}$ (Sarazin 1986), that is $\approx 2 \mathrm{Gyr}$ in the virialized cluster region ${ }^{3}$.

"Delayed quenching" models suggest that $\mathrm{S}$ can live in the cluster environment for several Gyr before being quenched (Wetzel et al. 2013; Fossati et al. 2017). The inferred timescales for morphological and PPS evolution from our and previous analyzes set an upper limit to the delayed quenching models timescale of 2-3 Gyr, and cannot exclude more rapidly quenching models (Oman \& Hudson 2016).

\section{Conclusions}

We use the WINGS data set to investigate the spatial and velocity distribution of cluster galaxies of three morphological classes, E, SO, and S. We do so by building stack clusters, using two different stacking normalization parameters, one based on velocity dispersion, the other on cluster richness. Our results do not depend on the stacking parameter. We build two stacks, one for regular (Reg) clusters and another for (Irr) clusters with significant evidence for substructures according to the DS test.

We determine the surface number density and velocity dispersion profiles $\Sigma(R)$ and $\sigma(R)$, of cluster E, S0, and S galaxies to an unprecedented accuracy. We fit $\Sigma(R)$ with both NFW and King models, and obtain marginally better fit results for the NFW model, independently of the morphological class and in both Reg and Irr clusters. We propose a new phenomenological model that provides acceptable fits to the $\sigma(R)$ of all our galaxy samples.

These are our main results:

- The projected phase space distributions of E, S0, and S galaxies are all different, both in Reg and in Irr clusters, at variance with a previous result (Biviano et al. 2002).

- The concentration, $r_{200} / r_{\mathrm{s}}$ or $r_{200} / r_{\mathrm{c}}$, increases from $\mathrm{E}$ to $\mathrm{S} 0$ to $\mathrm{S}$ galaxies, both in Reg and Irr clusters, reflecting the well-known morphology-radius relation (Sanromà \& Salvador-Solé 1990; Whitmore et al. 1993; Fasano et al. 2015).

- The concentration is higher in Reg than in Irr clusters for both E and S0 galaxies, but not for spirals.

- The velocity dispersion profiles have increasingly higher normalization and steeper slope for increasingly later-type

3 More precisely, the orbital time in a cluster is $\sqrt{8} \pi(\sqrt{\Delta} H)^{-1}$ $\left(\approx 8.9(\sqrt{\bar{\Delta}} H)^{-1}\right)$ for circular orbits and $\approx 7(\sqrt{\Delta} H)^{-1}$ for radial orbits (for NFW profiles, where $\Delta$ is the mean cluster overdensity relative to critical at the apocenter of the orbit). This leads to free-fall times from the virial radius of $2.1 \mathrm{Gyr}$ at $z=0.25$ ( $3 \mathrm{Gyr}$ ago) and $2.6 \mathrm{Gyr}$ now (for reasonably elongated orbits). 
galaxies (from $\mathrm{E}$ to $\mathrm{S} 0$ to $\mathrm{S}$ types), in agreement with the results of Adami et al. (1998), Biviano \& Katgert (2004).

- In Reg clusters, E and S0 galaxies have similar $\sigma(R)$, different from that of spirals; in Irr clusters, the S0 velocity dispersion profile is intermediate between those of the other two classes.

Our results indicate that the cluster environment is driving the transformation from infalling spirals to a dynamically more relaxed S0 population. In combination with previous results on the evolving fraction of cluster S0 galaxies with time (Postman et al. 2005; Smith et al. 2005; Desai et al. 2007), we argue that the timescale for this transformation is $\leq 3 \mathrm{Gyr}$. This transformation appears to be mostly completed in nearby Reg clusters, but still ongoing in nearby Irr clusters. We therefore expect S0s in Irr clusters to have lower ages on average than those in Reg clusters. Furthermore, we expect the PPSD of S0s in $z \gtrsim 0.4$ clusters, at a time where most S0s should be still evolving from infalling spirals, to be more similar to that of spirals than to that of ellipticals, unlike what we see in nearby clusters. Future investigations will be able to confirm or reject our expectations.

In future papers of this series we will use some of the results of this paper to perform a full dynamical analysis of the WINGS data-set, and to determine, in particular, the mass and velocity anisotropy profile of Reg WINGS clusters (Paper II: Mamon et al., in prep.) and their pseudo phase-space density profile (Paper III: Biviano et al., in prep.).

Acknowledgements. We wish to thank the anonymous referee for her/his use ful comments that have helped to improve this manuscript. The work of A.C. is supported by the STARFORM Sinergia Project funded by the Swiss National Science Foundation. This research has made use of the X-Rays Clusters Database (BAX) which is operated by the Laboratoire d'Astrophysique de Tarbes-Toulouse (LATT), under contract with the Centre National d'Études Spatiales (CNES). This research has made use of the NASA/IPAC Extragalactic Database (NED) which is operated by the Jet Propulsion Laboratory, California Institute of Technology, under contract with the National Aeronautics and Space Administration. A.B. acknowledges the hospitality of the Institut d'Astrophysique de Paris. The work of A.B. has been financially supported in part by the PRIN INAF 2014: "Glittering kaleidoscopes in the sky: the multifaceted nature and role of Galaxy Clusters", P.I.: Mario Nonino, by NSF grant AST-1517863.

\section{References}

Adami, C., Biviano, A., \& Mazure, A. 1998, A\&A, 331, 439

Arnaud, M., Pointecouteau, E., \& Pratt, G. W. 2005, A\&A, 441, 893

Balogh, M. L., Morris, S. L., Yee, H. K. C., Carlberg, R. G., \& Ellingson, E. 1999, ApJ, 527, 54

Bamford, S. P., Nichol, R. C., Baldry, I. K., et al. 2009, MNRAS, 393, 1324

Barnes, J. E. 1990, Nature, 344, 379

Bartelmann, M. 1996, A\&A, 313, 697

Beers, T. C., Flynn, K., \& Gebhardt, K. 1990, AJ, 100, 32

Bekki, K. 1999, ApJ, 510, L15

Bell, E. F., \& de Jong, R. S. 2001, ApJ, 550, 212

Biviano, A. 2011, in Proc. the Int. School of Physics Enrico Fermi - Course CLXXII - Astrophysics of Galaxy Clusters, eds. A. Cavaliere, \& Y. Rephaeli (Amsterdam: IOS Press), 95

Biviano, A., \& Girardi, M. 2003, ApJ, 585, 205

Biviano, A., \& Katgert, P. 2003, Ap\&SS, 285, 25

Biviano, A., \& Katgert, P. 2004, A\&A, 424, 779

Biviano, A., Katgert, P., Thomas, T., \& Adami, C. 2002, A\&A, 387, 8

Cava, A. 2008, Ph.D. Thesis, Department of Physics, Milano University
Cava, A., Bettoni, D., Poggianti, B. M., et al. 2009, A\&A, 495, 707

Desai, V., Dalcanton, J. J., Aragón-Salamanca, A., et al. 2007, ApJ, 660, 1151

D’Onofrio, M., Bindoni, D., Fasano, G., et al. 2014, A\&A, 572, A87

D’Onofrio, M., Marziani, P., \& Buson, L. 2015, Frontiers Astron. Space Sci., 2,

Dressler, A. 1980, ApJ, 236, 351

Dressler, A., \& Shectman, S. A. 1988, AJ, 95, 985

Dressler, A., Oemler, A. J., Couch, W. J., et al. 1997, ApJ, 490, 577

Fasano, G., \& Franceschini, A. 1987, MNRAS, 225, 155

Fasano, G., Poggianti, B. M., Couch, W. J., et al. 2000, ApJ, 542, 673

Fasano, G., Marmo, C., Varela, J., et al. 2006, A\&A, 445, 805

Fasano, G., Vanzella, E., Dressler, A., et al. 2012, MNRAS, 420, 926

Fasano, G., Poggianti, B. M., Bettoni, D., et al. 2015, MNRAS, 449, 3927

Fossati, M., Wilman, D. J., Mendel, J. T., et al. 2017, ApJ, 835, 153

Fritz, J., Poggianti, B. M., Bettoni, D., et al. 2007, A\&A, 470, 137

Fritz, J., Poggianti, B. M., Cava, A., et al. 2011, A\&A, 526, A45

Fritz, J., Poggianti, B. M., Cava, A., et al. 2014, A\&A, 566, A32

Fukugita, M., Shimasaku, K., \& Ichikawa, T. 1995, PASP, 107, 945

Ghigna, S., Moore, B., Governato, F., et al. 1998, MNRAS, 300, 146

Girardi, M., Biviano, A., Giuricin, G., Mardirossian, F., \& Mezzetti, M. 1993, ApJ, 404, 38

Groener, A. M., Goldberg, D. M., \& Sereno, M. 2016, MNRAS, 455, 892

Gullieuszik, M., Poggianti, B., Fasano, G., et al. 2015, A\&A, 581, A41

Gunn, J. E., \& Gott, J. R. 1972, ApJ, 176, 1

Jaffé, Y. L., Verheijen, M. A. W., Haines, C. P., et al. 2016, MNRAS, 461, 1202

Jing, Y. P. 2000, ApJ, 535, 30

Katgert, P., Mazure, A., Perea, J., et al. 1996, A\&A, 310, 8

Katgert, P., Mazure, A., den Hartog, R., et al. 1998, A\&AS, 129, 399

King, I. 1962, AJ, 67, 471

Lin, Y.-T., Mohr, J. J., \& Stanford, S. A. 2004, ApJ, 610, 745

Łokas, E. L., \& Mamon, G. A. 2001, MNRAS, 321, 155

Mahajan, S. 2013, MNRAS, 431, L117

Mamon, G. A., \& Łokas, E. L. 2005, MNRAS, 363, 705

Mamon, G. A., Biviano, A., \& Murante, G. 2010, A\&A, 520, A30

Mamon, G. A., Biviano, A., \& Boué, G. 2013, MNRAS, 429, 3079

Merrifield, M. R., \& Kent, S. M. 1989, AJ, 98, 351

Moore, B., Katz, N., Lake, G., Dressler, A., \& Oemler, Jr., A. 1996, Nature, 379, 613

Moran, S. M., Ellis, R. S., Treu, T., et al. 2007, ApJ, 671, 1503

Moretti, A., Poggianti, B. M., Fasano, G., et al. 2014, A\&A, 564, A138

Moretti, A., Gullieuszik, M., Poggianti, B., et al. 2017, A\&A, 599, A81

Moss, C., \& Dickens, R. J. 1977, MNRAS, 178, 701

Navarro, J. F., Frenk, C. S., \& White, S. D. M. 1996, ApJ, 462, 563

Neto, A. F., Gao, L., Bett, P., et al. 2007, MNRAS, 381, 1450

Oemler, A., Dressler, A., Kelson, D., et al. 2009, ApJ, 693, 152

Old, L., Skibba, R. A., Pearce, F. R., et al. 2014, MNRAS, 441, 1513

Oman, K. A., \& Hudson, M. J. 2016, MNRAS, 463, 3083

Omizzolo, A., Fasano, G., Reverte Paya, D., et al. 2014, A\&A, 561, A111

Pignatelli, E., Fasano, G., \& Cassata, P. 2006, A\&A, 446, 373

Poggianti, B. M., Aragón-Salamanca, A., Zaritsky, D., et al. 2009, ApJ, 693, 112

Postman, M., \& Geller, M. J. 1984, ApJ, 281, 95

Postman, M., Franx, M., Cross, N. J. G., et al. 2005, ApJ, 623, 721

Sanromà, M., \& Salvador-Solé, E. 1990, ApJ, 360, 16

Sarazin, C. L. 1986, Rev. Mod. Phys., 58, 1

Smith, G. P., Treu, T., Ellis, R. S., Moran, S. M., \& Dressler, A. 2005, ApJ, 620, 78

Sodré, L. J., Capelato, H. V., Steiner, J. E., \& Mazure, A. 1989, AJ, 97, 1279

Spitzer, L. J., \& Baade, W. 1951, ApJ, 113, 413

Stroe, A., Sobral, D., Dawson, W., et al. 2015, MNRAS, 450, 646

Tammann, G. A. 1972, A\&A, 21, 355

Thomas, T., \& Katgert, P. 2006a, A\&A, 446, 31

Thomas, T., \& Katgert, P. 2006b, A\&A, 446, 19

Valentinuzzi, T., Woods, D., Fasano, G., et al. 2009, A\&A, 501, 851

Varela, J., D’Onofrio, M., Marmo, C., et al. 2009, A\&A, 497, 667

Vijayaraghavan, R., \& Ricker, P. M. 2013, MNRAS, 435, 2713

Wainer, H., \& Thissen, D. 1976, Psychometrica, 41, 9

Wetzel, A. R., Tinker, J. L., Conroy, C., \& van den Bosch, F. C. 2013, MNRAS, 432,336

Whitmore, B. C., Gilmore, D. M., \& Jones, C. 1993, ApJ, 407, 489

Zabludoff, A. I., \& Mulchaey, J. S. 1998, ApJ, 496, 39 